\title{
Comments on: Optimization and data mining in biomedicine
}

\author{
Sergiy Butenko
}

Published online: 8 December 2009

(C) Sociedad de Estadística e Investigación Operativa 2009

In this review paper, Pardalos et al. discuss selected applications of operation research and system analysis in biomedicine. Some of the results presented in this paper, such as consistent biclustering and seizure prediction algorithms, are developed by Pardalos and his research group.

As for the material included in each of the sections, I believe that it is an excellent primer for young researchers who seek for introductory literature on applying optimization to solving problems in biomedicine.

The part on radiation therapy introduces the background on the application and the underlying optimization techniques with references to the medical instrumentation. Then models and solution approaches are presented and discussed.

The theoretical results discussed by Busygin et al. for consistent biclustering are of particular importance since, in general, there are not many papers discussing the theoretical foundations of biclustering.

The third application presents an alternative and innovative use of operations research in the electrode selection problem of EEG for improving the epileptic seizure prediction algorithm based on nonlinear dynamic time series analysis. This application shows how a formulation that is typically applied to problems of industrial interest can be modified and applied in a totally new framework.

The inclusion of EEG source localization problem in this survey is also a nice addition to the paper since the algorithms developed in this field are mainly based on signal processing approaches. An operations research point of view may give some more insight and new ideas for this inverse problem. This paper is a good reference paper for operational researchers and mathematicians and I believe it will be well received by the readers of TOP.

This comment refers to the invited paper available at: doi:10.1007/s11750-009-0124-1.

\footnotetext{
S. Butenko $(\bowtie)$

Department of Industrial and Systems Engineering, Texas A\&M University, College Station,

TX 77843-3131, USA

e-mail: butenko@tamu.edu
} 